University of Wollongong

Research Online

Faculty of Informatics - Papers (Archive)

Faculty of Engineering and Information

Sciences

$19-4-2004$

\title{
Arabic/Persian cursive signature recognition and verification using line segment distribution
}

\author{
A. Chalechale \\ University of Wollongong \\ G. Naghdy \\ University of Wollongong, golshah@uow.edu.au \\ Prashan Premaratne \\ University of Wollongong, prashan@uow.edu.au
}

Follow this and additional works at: https://ro.uow.edu.au/infopapers

Part of the Physical Sciences and Mathematics Commons

\section{Recommended Citation}

Chalechale, A.; Naghdy, G.; and Premaratne, Prashan: Arabic/Persian cursive signature recognition and verification using line segment distribution 2004.

https://ro.uow.edu.au/infopapers/52

Research Online is the open access institutional repository for the University of Wollongong. For further information contact the UOW Library: research-pubs@uow.edu.au 


\title{
Arabic/Persian cursive signature recognition and verification using line segment distribution
}

\author{
Abstract \\ This work proposes a fast method for line segment extraction based on chain code differentiation. It is \\ applied to cursive signature recognition of Arabic/Persian. The evaluation method is introduced to obtain \\ a quantitative value for the recognition rate. The comparative results show the existing differences among \\ the methods in recognition, building time and searching time criteria. The two methods used for \\ comparison are invariant moments and CBLSE method.

\section{Disciplines} \\ Physical Sciences and Mathematics

\section{Publication Details} \\ This article was originally published as: Chalechale, A, Naghdy, G \& Pramaratne, Arabic/Persian cursive \\ signature recognition and verification using line segment distribution, 2004 International Conference on \\ Information and Communication Technologies: From Theory to Applications, 19-23 April 2004, 475-476. \\ Copyright IEEE 2004.
}




\title{
Arabic/Persian Cursive Signature Recognition and Verification Using Line Segment Distribution
}

\author{
Abdolah Chalechale, Golshah Naghdy, and Prashan Pramaratne \\ School of Electrical, Computer \& Telecommunications Engineering, University of Wollongong \\ \{ac82,golshah,prashan\}@uow.edu.au
}

\section{Introduction}

There are several low-level image primitives used in computer vision and scene analysis. One of the most useful primitives is elementary line segments (ELSs). Line segment, as a primitive geometric object, is also used for face recognition [1]. Different characteristics of line segments distribution can serve as useful features in image retrieval applications. Chain code histogram is introduced in [2] and utilized for shape recognition of irregular objects.

English signature analysis, verification and recognition have been studied extensively. Arabic/Persian signatures, however, have different characteristics. While the former are often reshaped handwritten names, the latter are cursive sketches usually independent of the person's name. This paper proposes a new fast method for line segment extraction based on chain code differentiation. It is applied to cursive signature recognition. The results are compared with three other methods in terms of accuracy and speed.

\section{Line segment extraction and hybrid features (LSEHF)}

The input of the method is an edge map $I$ derived by an edge operator such as Canny on any real image. In addition, any thinned sketched image e.g. cursive signature can be used as the input $I$. First, the starting point of an 8-connectivity chain code is determined. The macro chain $A_{i}=\left\{a_{l}\right.$. $\left.a_{2} \ldots a_{n_{i}}\right\}, i=1,2 \ldots m$, where $m$ is the number of chains in $I$ and $n_{i}$ is the chain length, is obtained and put in a chain set $\left\{A_{i}\right\}$. For each $A_{i}$ we apply the following steps (see Fig. 1):

1. Noisy points, which make the chain over oscillating, are eliminated by median filtering .

2. The standard chain code representation has the wraparound drawback. To eliminate it, we produce a new modified code $B_{i}=\left\{b_{1}, b_{2} \ldots b_{n_{i}}\right\}$ for each $A_{i}=\left\{a_{1}, a_{2} \ldots a_{n_{i}}\right\}$ by a shifting operation [3] defined recursively as:

$\left\{\begin{array}{l}b_{1}=a_{1} \\ b_{i}=q_{i} \text { where } q_{i} \text { is an integer such that }\left(q_{i}-a_{i}\right) \bmod 8=0 \text { and is minimized for } i=1,2 \ldots n .\end{array}\right.$

3. The shifted chain code $B_{i}$ is then smoothed by a five-point Gaussian filter $\{0.1,0.2,0.4,0.2,0.1\}$. Let $\Gamma(\theta)$ be the shifted and smoothed waveform where $\theta$ is the traversing variable.

4. The extreme points of $d \Gamma / d \theta$ are considered as break points $\left(\xi_{i}\right)$, if they are greater than a threshold.

The line segment $l_{i}$, which connects $\xi_{i}$ to $\xi_{i+l}$, is considered as the lineal approximation of the micro chain lying between the two points. The total collection of $l_{i}$ 's, say $L$, is the line segment set of the image/sketch $I$.

The lengths of the line segments in $L$ are rotation and translation invariant. Normalizing the length by accumulating length makes it scale invariant as well. Furthermore, the corner angel between adjacent segments is scale, rotation and translation invariant. 
For the purpose of cursive signature recognition, we employ these two principles to extract two discriminating and affine transform invariant vectors. The vectors are made as follows.

1. For each segment in $L$, compute the length and normalize it with accumulative length.

2. For each adjacent segment pair compute the corner angel.

3. Uniformly quantize the normalized length and corner to $U$ and v equal parts.

4. Construct two vectors and count the number of line segments and the number of corners in each entry according to the normalized length and the angle.

\section{Experimental results}

We assumed an office with 70 employees and collected 70 Arabic/Persian cursive signatures (set $P)$. Each signature in $P$ was scanned with $300 \mathrm{dpi}$ and then morphologically thinned. The feature vectors were obtained off-line for each signature using the proposed method with $U=40$ and $V=36$. $\mathrm{We}$, then collected signatures from the same persons at another time (set $Q$ ) and the vectors were obtained through the same process. To evaluate the accuracy of the proposed method, we applied all signatures in $Q$ as queries while regarding $P$ as signature models. Fig. 2 shows some signatures in our test data.

We also constructed a 64-bin chain code histogram, which is an extension of the 8-bin chain code histogram proposed in [2]. Another two methods used for comparison are invariant moments and CBLSE method [4]. We used the evaluation method introduced in [5] to obtain a quantitative value for the recognition rate. The comparative results show the existing differences among the methods in recognition rate, building time (off-line), and searching time (on-line) criteria. The proposed method outperforms the others in recognition rate while in off-line and on-line speeds is similar to or better than the others.

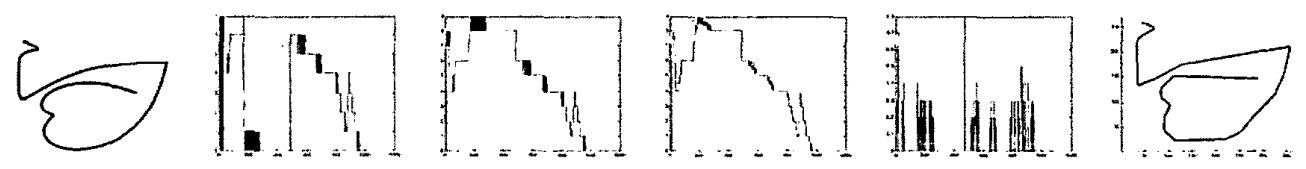

Fig. 1 From left to right: an example contour, median filtered chain code, shifted, smoothed, derivative representations, and extracted line segments

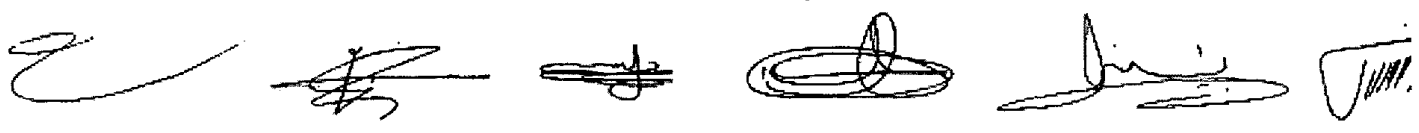

Fig. 2 Cursive signature examples

\section{Refrences}

1. Yongsheng, G. and Leung, M.K.H. Face recognition using line edge map.

2. Livarinen, J. and Visa, A. Shape recognition of irregular objects.

3. Hui, L., Manjunath, B.S. and Mitra, S.K. A contour-based approach to multisensor image registration.

4. Chalechale, A. and Mertins, A. Line segment distribution of sketches for Persian signature recognition.

5. Chalechale, A. and Mertins, A. An abstract image representation based on edge pixel neighboring information. 